\title{
ICT to Go Kaput due to Impending Solar Flare: Some Counteractive Measures
}

\author{
Mohammad Talib \\ Department of Computer Science, University of Botswana \\ Gaborone, BOTSWANA
}

\begin{abstract}
The sun is pretty much a big nuclear reactor. A solar storm is when wisps of that material go outside of the sun that is highly charged with electromagnetic energy so it screws up our electronics. When the earth's magnetic poles switch there will be a period of time where the earth's magnetic field doesn't protect us from those charged particles and all of our electronic technology will be fried. Bright flash of plasma in the Sun's atmosphere, capable of affecting radio traffic on Earth when particles of the plasma arrive at the Earth, Flares are most common at peak periods for sunspot activity, and are accompanied by ultraviolet and X-ray emissions as well as bright flashes of light. Flares seem to operate by a mechanism in which energy is stored by the Sun's magnetic field and then released in a single sudden burst. If a powerful solar flare struck earth, it could destroy crucial technology, mainly the ICT, and also cause a worldwide blackout. NASA has issued a warning of such massive sun solar flare storm to hit earth with force of $100 \mathrm{~m}$ hydrogen bombs in 2012. Here, in this paper a worst case scenario of this storm, if it happens, has been discussed with its pros and cons. Also the paper discusses the some present and future counteractive measure in the event of such happening.
\end{abstract}

\section{General Terms}

Information and Communication Technology, Astro Physics, Information Technology, Satellite Science

\section{Keywords}

CME, Solar Storm, Aurora, Radiation, Flares, Sun, Solar Wind, X-ray, NASA, Plasma, Sunspot, Earth, Particles, Light, Field, ICT, Communication, Internet, Satellite

\section{INTRODUCTION}

\subsection{Information and Communication Technology}

ICT (Information and Communication Technology) is similar to IT (Information Technology) ${ }^{[21]}$ but focuses more on telecommunications mediums. Information and communication have been advancing very fast. Today ICTs are generally regarded as the driving force behind our daily life. ICTs include all communication devices or applications, electronic networks services including hardware applied through networks services ${ }^{[1]}$. These include radio, television, mobile phone, internet, software systems, hardware, computing information services, multimedia, telephone, fax, and electronic news. Modern forms of ICT have made it possible for users across the world to communicate with each other in real-time on a regular basis ${ }^{[21]}$.

ICT covers any product that will store, retrieve, manipulate, transmit or receive information electronically in a digital form ${ }^{[10][5]}$. For example, personal computers, digital television, email, robots etc. So, we can say that the ICT is concerned with the storage, retrieval, manipulation, transmission or receipt of digital data ${ }^{[6]}$. Importantly, it is also concerned with the way these different uses can work with each other. In business, ICT is often categorized into two broad types of products - one is the "traditional computer-based technologies"; where we can typically do the things on a personal computer or using computers at home or at work and the other more recent and fastgrowing range of "digital communication technologies" which allow people and organizations to communicate and share information digitally ${ }^{[7]}$. The $\mathrm{C}$ part of ICT refers to the communication of data by electronic means, usually over some distance. This is often achieved via networks of sending and receiving equipment, wires and satellite links.

\subsection{Solar Storm and Solar Flare}

A solar storm is an event in which activity on the Sun interferes with the Earth's magnetic field. Solar storms occur as a result of events such as solar flares and Coronal Mass Ejections $(\mathrm{CME})^{[2]}$. These events generate a so-called "solar wind," a gust of charged particles which can slam into the Earth's magnetic field in hours if the solar wind happens to be traveling in the direction of Earth. The collision can generate quite a light show as a geomagnetic storm erupts, and the storm can last for several days under the right conditions.

A solar flare is a sudden brightening observed over the Sun surface or the solar limb, which is interpreted as a large energy release of up to $6 \times 10^{25}$ joules of energy ${ }^{[14]}$ (about a sixth of the total energy output of the Sun each second). The term is also used to refer to similar phenomena in other stars, where the term stellar flare applies. In general terms, a solar flare is a violent explosion in the Sun's atmosphere with an energy equivalent to tens of millions of hydrogen bombs. Solar flares take place in the solar corona and chromospheres, heating the gas to tens of millions Kelvin and accelerating electrons, protons and heavier ions to near the speed of light. They produce electromagnetic radiation across the spectrum at all wavelengths from long-wave radio signals to the shortest wavelength gamma rays. 


\section{SCOPE OF THE PAPER}

Based on the introductory notes, one on the ICT and the other on the impending solar flare in the days ahead, this paper provides links between the two, giving glimpses of a wide variety of research and action regarding ICTs and its uses in the society. Such links include human development as part of provisions all across the globe to improve the lives of citizens, universal access based on pricing of the information communications technologies and services offered and ICT innovation for service delivery, all of which have the potential to lead to make the communication fast, easy and cheap. The entire ICT is based on the internet and a satellite is the main communication link to establish the status of the ICT. Because the Sun is so far away, many people believe that solar storms are not capable of causing very much damage, but they can in fact be quite devastating. Research in the early 2000 s created a number of potential worst case scenarios which illustrated the destructive power of solar storms, including scenarios showing that powerful nations in the developed world could be brought to their knees for a decade or more by a storm of large magnitude.

The impending solar flare is going to be a big threat to the ongoing communication link as it has been predicted that the solar storm which is taking shape in the coming years from 2012 that will release solar flares and some of them may reach up to the earth by destroying all communication links on its pathways. This paper seeks to look into the data from literature and other sources especially current reports from various research agencies including NASA on the impact of solar flare on the currently used ICT devices and suggests some remedial steps for now and future. Literature has then been reviewed to find out the impact of the solar flare on the ICTs and their effects. Recommendations have been proposed at the end of the paper that how to take counteractive measures to minimize the effect of the solar flare on the currently used ICTs. While the paper seeks to inform on the impact of solar flare on ICT, its other prediction of the impending solar flare are going to be grievous.

\section{LITERATURE REVIEW}

Solar flares were first observed on the Sun in 1859 by English astronomer Richard Carrington ${ }^{[3]}$. Similar stellar flares have also been observed to varying degrees on other stars in modern times. The frequency of solar flares varies, from several per day when the Sun is particularly "active" to less than one each week when the Sun is "quiet". Solar flares may take several hours or even days to build up, but the actual flare takes only a matter of minutes to release its energy. Solar activity is classified as A, B, C, M or X according to the brightness of its X-rays near Earth as measured on the GOES spacecraft in Watts per square meter $\left(\mathrm{W} \mathrm{m}^{-2}\right)$. Each class is ten times more powerful than the preceding one, with $\mathrm{X}$ at $10^{-4} \mathrm{~W} \mathrm{~m}^{-2}$ ). Within a class there is a linear scale from 1 to 9 , so an $\mathrm{X} 2$ flare (twice as powerful as an X1 flare) is four times more powerful than an M5 flare (five times as powerful as an M 1 flare). Solar activity is normally within the $\mathrm{A}$ to $\mathrm{C}$ range. Class $\mathrm{C}$ flares have little effect on Earth, while the more powerful $M$ and $\mathrm{X}$ flares can cause disruption and damage. Flares generally stay below $\mathrm{X} 10$, but infrequently $\mathrm{X}$ designations run 'off the charts'. X20 events that were recorded on August 16, 1989 and April 2,2001 were outshone by a flare on November 4, 2003 that was the most powerful X-ray flare ever recorded: an X28. Sunspot Region 486, where this flare originated was the most turbulently active sunspot ever record ed ${ }^{[4]}$.

It was long thought that solar flares send out streams of highly energetic solar wind that can present a radiation hazard to spacecraft outside of a planetary magnetosphere and can disrupt radio signals on Earth. They were also thought to be a primary contributor to the aurora borealis and aurora australis and to solar proton events ${ }^{[13]}$. However, it is now thought that Coronal Mass Ejections (CMEs) that often, but not always, accompany flares, are the main cause of such effects on and around the Earth. The radiation risk posed by solar flares and CMEs is one of the major concerns in discussions of manned missions to Mars. Some kind of physical or magnetic shielding will be required to protect the astronauts.

\subsection{The Sun and Inside It}

The Sun seems to be a white-hot disk with an extremely sharp edge, but we know that it is spherical in form. Although the Sun seems uniform but telescopic view shows it to be strongly textured (Fig.1). The most obvious features are the sunspots, dark cool regions surrounded by intensely hot solar gases. The Sun changes daily and it is full of my steries apart from sunspots. The centre of the Sun is 2.5 times brighter than the edge. The reason for this variation is quite simple. Rays from the centre of the disk come vertically from the surface. Rays from near the edge travel along highly inclined paths. The Sun loses heat by radiation and this must be replenished continuously by a flow from within.

The sun is made of gas, nothing but gas ${ }^{[17]}$. Like all other stars, the Sun is evolving. About 5 billion years ago it formed from a large cloud containing almost pure hy drogen gas and dust. The cloud contracted under its own gravitational forces, losing gravitational energy in doing so and converting it to heat. The geological evidence shows that the earth is about 4 billion years old, and the sun is little older. The sun still contains enough hydrogen fuel to maintain its present output for another 10 billion years. Sun is highly ionized, and the free electrons make it an extraordinarily good electrical conductor. Radiant heat flow is the dominant transport mechanism throughout most of the Sun. Radiation is emitted when hot electrons collide with atoms. The radiation travels at the speed of light (300, $000 \mathrm{~km} / \mathrm{s}$ ) until absorbed. Although in free space radiation would travel a distance equal to the Sun's radius in little more than 2 seconds, absorption is so strong inside the Sun that it takes 1 million to 2 million years to diffuse out. Thus, the light and warmth we receive were produced near the Sun's centre over a million years ago. 


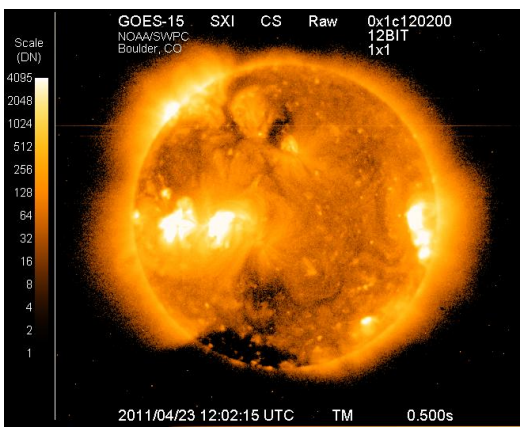

Fig.1: The latest picture of the Sun taken on 23/04/2011 at 12:02:15 UTC/GMT

Source: NOAA (National Oceanic and Atmospheric Administration) USA

\subsection{Significance of flares and their effects}

Flares are happenings. As late as 1960, we would have called them chromospheric flares ${ }^{[2]}$. Small flares may last only a few minutes, big flares an hour or more. Flares on the sun (Fig.2) were quite unsuspected before 1859. The largest radiation storm of solar flares since October 1989 occurred in January 22, 2005 as pictured in Fig. 3. Today, although flares may a source of wonder and delight, their effects are a source of concern to scientists, to industries, to governments and above all the current ICTs. Considerable effort goes into predicting the effects of flares on human activities on earth and in space. The following are the descriptions of the most significant effects of flares follow: ${ }^{[18]}$

X-rays enhance the electron density in the ionosphere, disturbing radio communications. A radio wave propagating in the ionosphere is absorbed or refracted, depending upon the wave frequency. One result of the enhanced electron density can be immediate and sudden loss of short-wave radio communication that can last an hour or more. Another is an abrupt change in transmission path of navigational signals, which can lead to erroneous determination of location.

Energetic protons damage electronic components of detectors, transducers, and ICTs in satellites. They endanger humans in space and in stratospheric flights over the poles, where protection of the geomagnetic field is diminished. They enhance the ionization density of the polar ionosphere, resulting in increased absorption of highfrequency waves propagated into that region.

The geomagnetic field can change abruptly due to a variation of the magnetic field and momentum of the solar wind impinging on earth's magnetosphere. This disturbs the ionosphere, and affects measurements used in geophysical extrapolation. Induced current can disrupt electric power transmission and cable communications and affect oil pipelines. Heating of the upper atmosphere associated with geomagnetic disturbances changes its density structure, increases atmospheric drag on satellites and thwarts efforts to predict satellite orbits.

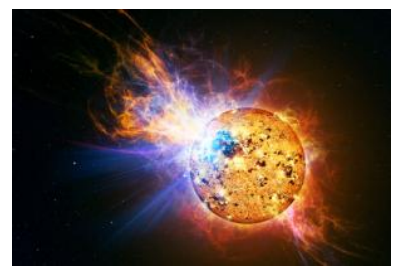

Fig.2 Latest images for the solar flares Source: Universe Today

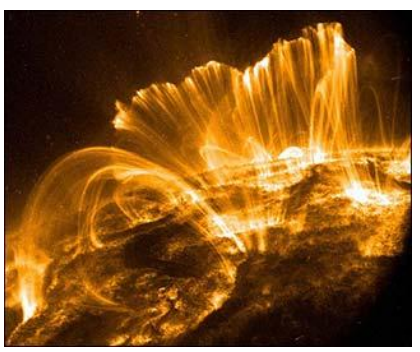

Fig.3 Largest Radiation S torm of S olar Flares Since October 1989 Occurred in January 22, 2005 Source: NASA,. USA

\subsection{Solar Flare Prediction}

In order to predict a solar flare two areas have so far been investigated: the observations, analysis, and theories aimed at understanding the flare process, and the practical operations of gathering and interpreting relevant data and making and distributing the flare forecast ${ }^{[22]}$. A search of the literature included unpublished reports and proceedings of meetings and workshops as well as research papers and reviews in scholarly journals and books.

Despite rapid progress, flare physics is still far from attaining the exact and quantitative understanding of flare processes required for accurate prediction but there is a prediction for 2012 (Fig. 4). On the other hand, most flares occur in active regions (Fig. 5), and most big flares occur in strong, complex magnetic fields; when an endeavor is sensitive to flare occurrence, an experienced forecaster with current information on solar activity can offer a great deal of guidance. But how much can be expected? How good are the forecasts now? This is another question that lacks a clear answer at present. The route for evaluating forecasts is not well marked but is at present a tangle of nonstandardized terms, differing objectives, and reluctance to compare the skills of individual forecasters.

Current methods of flare prediction are problematic, and there is no certain indication that an active region on the Sun (Fig. 5) will produce a flare. However, many properties of sunspots and active regions correlate with flaring. For example, magnetically complex regions (based on line-ofsight magnetic field) called delta spots produce most large flares. A simple scheme of sunspot classification due to McIntosh is commonly used as a starting point for flare prediction. Predictions are usually stated in terms of probabilities for occurrence of flares above $\mathrm{M}$ or X GOES class within 24 or 48 hours. The U.S. National Oceanic and 
Atmospheric Administration (NOAA) issues forecasts of this kind ${ }^{[19]}$.

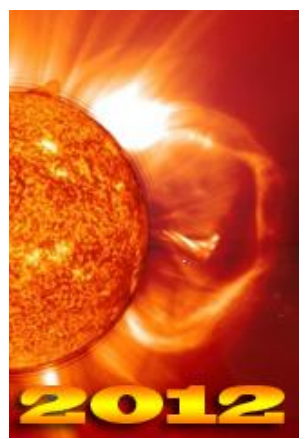

Fig.4 Prediction of solar flares Source: NASA, USA

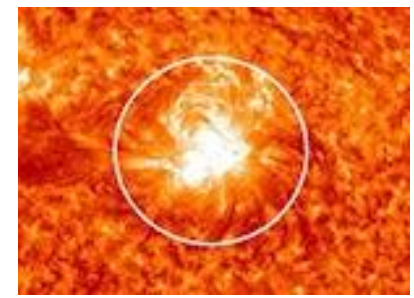

Fig.5 Solar Activity Heating-up Picture taken by NASA on 14/04/201 1

\subsection{Interaction with the planetary processes}

The solar wind also carries with it the magnetic field of the Sun. This field will have either a North or South orientation (Fig.6). If the solar wind has energetic bursts, contracting and expanding the magnetosphere, or if the solar wind takes a southward polarization, geomagnetic storms can be expected. The southward field causes magnetic reconnection of the dayside magnetopause, rapidly injecting magnetic and particle energy into the Earth's magnetosphere. During a geomagnetic storm, the ionosphere's $\mathrm{F}_{2}$ layer will become unstable, fragment, and may even disappear. In the northern and southern pole regions of the Earth, auroras will be observable in the sky.

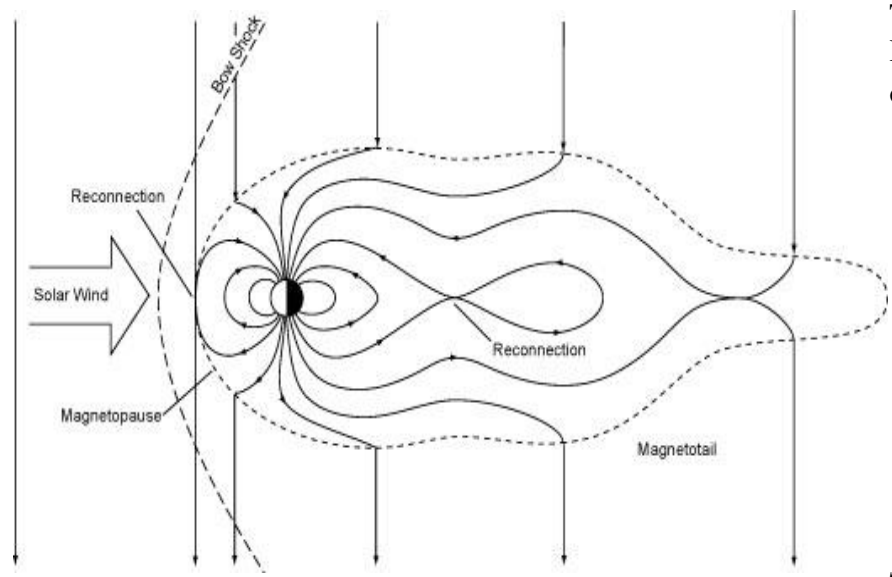

Fig.6 Magnetic field of the sun due to solar wind Source: Wikipedia

\section{HISTORICAL OCCURRENCES}

From August 28 until September 2, 1859, numerous sunspots and solar flares were observed on the sun, the largest flare occurring on September 1. A massive CME headed directly at Earth due to the solar flare and made it within eighteen hours - a trip that normally takes three to four days. On September $1-2$, the largest recorded geomagnetic storm occurred. The horizontal intensity of geomagnetic field was reduced by $1600 \mathrm{nT}$ as recorded by the Colaba observatory near Bombay, India. Telegraph wires in both the United States and Europe experienced induced emf, in some cases even shocking telegraph operators and causing fires. Auroras were seen as far south as Hawaii, Mexico, Cuba, and Italy phenomenon those are usually only seen near the poles. This was the 1859 solar superstorm. On March 13, 1989 a severe geomagnetic storm caused the collapse of the Hydro-Québec power grid in a matter of seconds as equipment protection relays tripped in a cascading sequence of events. ${ }^{[4][1]}$ Six million people were left without power for nine hours, with significant economic loss ${ }^{[15]}$. The storm even caused auroras as far south as Texas. ${ }^{[8]}$ The geomagnetic storm causing this event was itself the result of a CME, ejected from the Sun on March $9,1989^{[9]}$. Ice cores show evidence that events of similar intensity recur at an average rate of approximately once per 500 years. Since 1859 , less severe storms have occurred in 1921 and 1960, when widespread radio disruption was reported [3]. In August 1989, another storm affected microchips, leading to a halt of all trading on Toronto's stock market ${ }^{[20]}$. Since 1989, power companies in North America, the UK, Northern Europe and elsewhere evaluated the risks of geomagnetically induced currents (GIC) and developed mitigation strategies. Since 1995, geomagnetic storms and solar flares have been monitored from the Solar and Heliospheric Observatory (SOHO) joint-NASA-European Space Agency satellite. On February 26, 2008 the magnetic fields erupted inside the magnetotail, releasing about $10^{15}$ Joules of energy. The blast launched two gigantic clouds of protons and electrons, one toward Earth and one away from Earth. The Earth-directed cloud crashed into the planet below, sparking vivid auroras in Canada and Alaska ${ }^{[16]}$. The image of aurora (Fig. 7) was captured from the International Space Station in May 2010 when it currently occurred over the southern Indian Ocean.

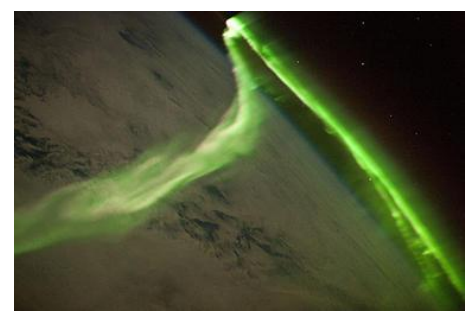

Fig.7 Aurora over the southern Indian Ocean Source: A photo taken from the Intemational Space Station in May 2010 


\section{A WORST CASE SCENARIO}

A study by the ISES (the International Solar Energy Society) on the solar cycle sunspot number progression has been done and they predicted the completion of solar cycle 24 somewhere in 2012 which will be at its peak in 2013 as given in the graph in Fig. 8 and will continue upto 2017 when the sun will release a number of flares which are likely to cause havoc on the earth. Also the study which was done in 2009 by the National Academy of Sciences which predicts that a solar storm will be arriving in 2012 which would be similar to the one in 1859 that shut down telegraph lines all over the USA, NASA recently discovered that the solar storm could shut down the United States for months. Sir John Beddington, the British Government's chief scientist, said that the Sun was waking up from a quiet period and was likely to throw a lot more "space weather" at the Earth. Also, the world was increasingly vulnerable to damage because of our dependence on satellites, communication networks and computer devices. If a solar storm hit the Earth, it could throw the entire ICT out of gear that would lead to complete paralysis to communication and other related equipments and their usage. This would also throw out navigation systems, crash stock markets, ground aircraft and cause power cuts. Transformers could get wiped out, meaning that whole continents would not have electrical power. Gone too would be the satellites controlling most of our luxuries today means that your refrigerator and freezer wouldn't have any electricity, leaving food to rot and creating food shortages around the world. Communication systems and power systems worldwide could be wiped out for months at a time. Something this large would cause not just a localized but rather a continent-wide power outage. Food riots would break out, governments could collapse... and there would be no cavalry coming to the rescue because the cavalry would also lose its power and ability to communicate. ${ }^{[19]}$ Lawrence E. Joseph, a disaster expert, states that these solar storms which will hit in 2012 will potentially effect power grids, satellites, and cost trillions daily for the world. He has written a book, "Guilty of Apocalypse: The Case Against 2012", in which he says that the year 2012 will be "pivotal, perhaps catastrophic, possibly revelatory, to a degree unmatched in human history". More storms will be frequent by the summer of 2011 until 2017 as per the study of the graph in Fig. 8.

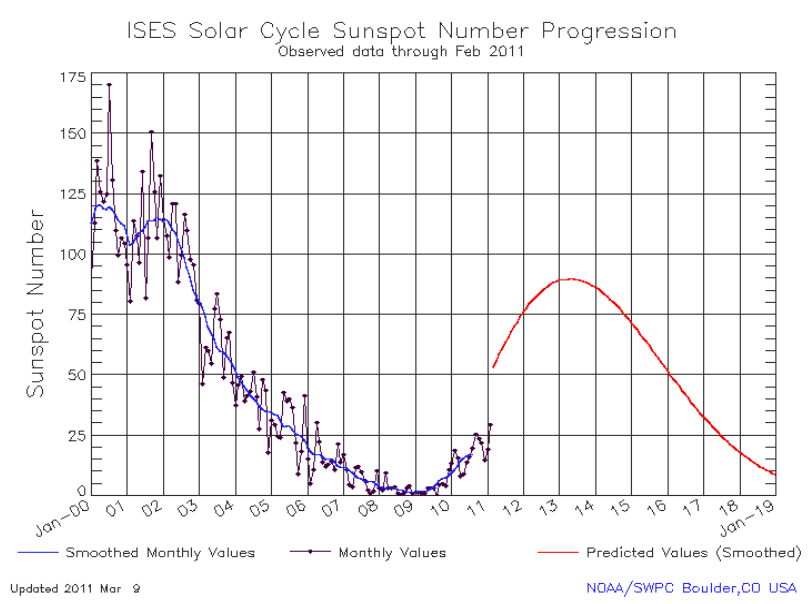

Fig. 8S olar Cyde Sunspot Number Progression Source: National Aeronautics and Space Administration (USA)

If a super solar storm slams into the earth, this no doubt will trigger an international crises and Martial Law ${ }^{[19]}$ will follow soon afterwards. There is a provision of such Martial Law in the US when the country has been facing some national or civil crisis. So if you are in the Unites States, whatever you do, you want to make sure you not only get home once the super solar storm starts, but you want to make sure that you continue to stay at home until after the solar storm is over and only after Martial Law is over as well. The solar storm could last for weeks or may be months. In that case you need anywhere from 12 to 24 months of supplies.

\section{Some Counteractive Measures}

Every eleven years the sun flips with respect to its magnetic field (North becomes South and South becomes North) and releases a burst of radiation directed toward outer space, including Earth, and that this burst could endanger our communication systems. As of now the report, however, is careful to say that this may in fact not happen. It's inevitable, however, that at some time or another it will happen again. We, therefore, need to take some counteractive measures for future. For example, even though satellites currently in outer space cannot be reinforced, the next generation of satellites should be reinforced to withstand the full brunt of the solar winds and solar flares. Also, on Earth, power stations and especially transformers should be insulated and reinforced so as to be able to withstand radiation coming from outer space. This is not rocket science; we know how to do it and the technology is already there-it's just a question of political will.

The following was one of the best Yahoo! answers to the question about the possible personal protection against solar flare radiation which is evident: The best way to protect yourself would be to put something reflective on your head to block incoming radiation and follow these precautions in order to survive the coming onslaught:

- Batteries: Turn anything running on batteries upside down. This will "shift calibrate" all of your D.C. power sources. Remove the battery from your car and place it 
in an aluminum lined box (tin foil will do). This will block all rays and will keep it from exploding. DONOT TURN IT UPSIDE DOWN. Car batteries operate on a different principle than your regular batteries. Simply putting it in the box should be all the protection that is needed.

- Wear highly reflective clothing Paint a jumpsuit or pair of ordinary coveralls with chrome colored paint, and wear a hat covered with foil. This will protect your body from these rays.

- Shield your eyes Paint an ordinary pair of plastic sunglasses with the same paint. Scratch slits on the lenses to allow y ou to see through them.

Here are some of the bulleted suggestions to protect your ICT devices from the solar storm in future ${ }^{[12]}$ :

- Use a UPS on your computer and TV

- Install lightning and surge arrestors

- Use power strips on computers and TV's

- Wire solar energy systems to voltages lower that 150 volts

- Go off the grid

Besides these, some more ICT preventative measures exist, including digging transmission cables into the soil, placing lightning rods on transmission wires, reducing the operating voltages of transformers, and using cables that are shorter than $10 \mathrm{~km}$.

\section{CONCLUSION}

Solar storms will continue to take place and due to that the impending solar flare in the years ahead, if not today, is going to cause a big destruction on the earth for sure. What has been done, so far in the ICT area, cannot be undone but in future the preventive measures can be taken - satellites can be sent offline during big flares, power grids and communication networks can be shielded against electromagnetic radiation and so on. But it is not the end of the world, for sure.

\section{REFERENCES}

[1] Adeya, N.: Information and communication technologies in development. In: Global Equity Conference, Rethinking ICTs in Africa, Asia, and Latin America, Heerlen Maastrisht, Nertherlands, 2002

[2] Alex G. Smith, Radio Exploration of the Sun, D Van Nostrand Company Inc., 1967

[3] Bracing the Satellite Infrastructure for a Solar Superstorm, Sten F. Odenwald and James L. Green, July 28,2008

[4] Canadian Broadcasting Corporation (CBC News) 22 October 2005

[5] Clarke, A., Englebright, L. ICT: The new basic skill. Niace, Leicester, 2003
[6] Doyle, S,. Information and Communication Technology: Vocational A level. Nelson Thornes Ltd, Cheltenham, 2001

[7] Duff, A, Impact of ICT on society. In: J Lawson (ed.), $\mathrm{J}$, : Information and communication technology: options, Pearson Education Limited, Essex, 2002

[8] Earth dodges magnetic storm, New Scientist, Magazine Issue, 24 June 1989

[9] Earth in Space (American Geophysical Union) 9 (7): 9-11. March 1997

[10] Gehris, D.O., Szul , F.L.: Communications Technologies., Prentice Hall, Upper Saddle River, 2002

[11] Geomagnetic Storm http://en.wikipedia.org/wiki/Geomagnetic_storm\#CIT EREFBolduc 2002 date of acess $23^{\text {rd }}$ April 2011

[12] How to protect yourself from the next solar or lightening storm http://www.examiner.com/ date of access $23^{\text {rd }}$ April 2011

[13] Jun-Ichi Sakai and Cornelis De Jager, Solar Flares and Collisions Between Current- Carrying Loops, Kluwer Academic Publishers, Kluwer Academic Publishers, The Netherlands, 1996

[14] Kopp, G.; Lawrence, G and Rottman, G. (2005). "The Total Irradiance Monitor (TIM): Science Results" Solar Phy sics (2005) 230: 129-139 Springer 2005

[15] Lawrence E. Joseph: Guilty of Apocalypse: The case Against 2012, Amazon.com, USA, ISBN: 978-0-76792448-1, 2007

[16] Plasma Bullets Trigger Northern Lights Jeff Haperman, NASA Science, Science News, July 2008

[17] Ronalrd G. Giovanelli, Secrets of the Sun, Cambridge University Press, Cambridge, 1984

[18] Sawyer, C., Warwick, J.W. and Dennett, J.T. : Solar Flare Prediction, Colorado Associated University Press, Colorado, 1986

[19] Solar Storm Wrning http://www.solar-stormwarning.com date of access $23^{\text {rd }}$ April 2011

[20] Solar storms halt stock market as computers crash, 9 September 1989 by Leigh Dayton, Vancouver Issue 1681

[21] The Sharpened Glossary, Definitions of Computer and Internet Terms, http://www.sharpened.net/glossary/definition/ict date of access $23^{\text {rd }}$ April 2011

[22] Yoshimori M.: Symposium on Current-Loop Interaction in Solar Flares Toy ama University, p.45 in J.I.(ed.), 1994 